\title{
Waist circumference disparity: a comparison of American, European and Asian cut offs among Africans
}

\begin{abstract}
Introduction: The world is a constellation of Homo sapiens with different orientation to life. This disparity in orientation had been of genetic, physiologic and phenotypic importance even before the times of Hippocrates. Due to phenotypic differences in waist circumference, the Americans, Europeans and Asians have different scientifically generated cut off values for people of their origin. The same cannot be said of Africans.

Aim: The aim of this study is to analyse the disparity in applying the American, European and Asian cut offs to Africans.

Methods: This preliminary prospective study was carried out in Nigeria. The umbilicus was used as the land mark for measurement of waist circumference using a non stretch tape.

Results: The population of this cohort was 126 . The age range was 18-88years. By using the American cut off, $68.25 \%$ of the cohort was normal. By using the European cut off $43.65 \%$ of the cohorts were normal. By using the Asian cut off $43.65 \%$ of the cohort studied were normal.
\end{abstract}

Discussion: The disparity in using the different cut off for Africa is glaring.

Conclusion: Africans need to research and generate an indigenous African descent based cut offs for better prediction of cardiovascular risk amongst Africans.

Keywords: waist circumference disparity, American, Asian, Africans, BMI, WC
Volume 4 Issue 3 - 2017

\author{
Ademolu Adegbenga Bolanle \\ Department of Medicine, Lagos State University Teaching \\ Hospital, Nigeria
}

Correspondence: Dr Ademolu Adegbenga B, Department of Medicine, Lagos State University Teaching Hospital, I-5, Oba Akinjobi way, lkeja GRA, Lagos, Nigeria, Tel +2348033575940, Email ademoluab@yahoo.com

Received: July 24, 2017 | Published: December 18, 2017

\section{Introduction}

The world is a constellation of Homo sapiens with different orientation to life. This disparity in orientation had been of genetic, physiologic and phenotypic importance even before the times of Hippocrates. The diversity of the universe, human race and mankind is not only in socioeconomic, political or cultural value, it also involves diversity in physiology of disease pattern and by extension diagnostic and management pattern. While some part of the universe are advanced, some are not and the World Health Organisation and World Bank based on this amongst other consideration has classified countries as low middle and high income countries. ${ }^{1}$

Due to phenotypic differences in waist circumference, different continent of the world notably Americans, Europeans and Asians have different but appropriate scientifically generated cut off values for people of their origin or descent since Waist Circumference (WC) is a cardiovascular risk factor associated with hypertension, diabetes mellitus and dyslipidemia., ${ }^{2,3}$ The same cannot be said of Africans, there is no normal acceptable indigenous cut off value for waist circumference for Africans as compared to their American, European or Asian counterpart.

It therefore follows that for scientific and reference purposes, Africans as a whole for now have to adopt other continents cut off numbers as normal for people of African race or descent. This raises these questions are their differences in applying other races cut offs to Africans or not? Is there gender variability in the application of other races cut off to Africans? The three other races mentioned above have different cut offs which has been defined for their race. Africa, which makes up $22 \%$ of the world's total land area, is the second largest continent. Culturally, Africa is diverse, with an estimated population of 642 million living in less than 50 countries and making up 3000 ethnic groups and greater than or equal to 1000 languages. It is very much part of the developing world. Indeed sub-Saharan Africa is in the middle of one of the most rapid demographic and epidemiologic transitions in world history, and different countries and regions are at different stages of this transition. ${ }^{4}$

Using Nigeria, the most populous black African country as a case study, the cut off of our colonial master country is widely adopted as the cut offs for defining normal waist circumference. But let me quickly point out that just as westernisation and western life among Africans is bringing about an increase in prevalence of diseases of the western world among Africans ${ }^{4}$ the same way, applying Europid cut offs or any other cut off to Africans without an extensive African based research giving African cut offs, will result in diagnostic error of varying degree since the sensitivity, specificity and predictive value of other races cut offs will differ among Africans compared to the original race where the cut offs was generated from.

The aim of this interesting study is to analyse the disparity in applying the American, European and Asian waist circumference cut offs to Africans. It is hoped that reading this article will not only sensitise but also challenge and awake Africans to unite and get a continentally acceptable cut offs for waist circumference. 


\section{Methodology}

This preliminary prospective study was carried out in Nigeria, the most populous black African nation in the city of Lagos. The waist circumference of each individual was measured following obtained consent from each participant from the general African population during a cross sectional survey. All measurements were taken during a free medical screening programme in Nigeria. The umbilicus was used as the land mark for measurement using a non stretch tape held snugly to the body parallel to the floor, at normal respiration with the participants standing upright with arms by the sides and feet close together and weight evenly distributed across the feet. ${ }^{5}$

None of the participants ate at least four hours to the measurement. The exclusion criteria used were pregnancy, ascites, abdominal mass from any cause, anasarca, puerperium. Demographic data were obtained excluding name. The reading for each individual was recorded. The limitation of the study is cardiovascular risk factors (diabetes, hypertension, dyslipidemia) were not assessed with the aim of correlating it with large waist circumference in the cohort studied. Also in this preliminary study only a small Nigerian population was used but a large multinational African study size is suggested in the future in order to get a scientific value for Africans in general.

\section{Results}

The population of this cohort was 126 . Of these male formed $40.48 \%$ (51 participants), while female formed $59.52 \%$ (75 participants). The age range among the cohort was $18-88$ years. The age range among male was 23-72years with male mean age of 48.84 years. The age range of female was 18-88years with female mean age of 46.47years. The mean age of the general population was 47.43 years.

By using the American waist circumference cut off of $>102 \mathrm{~cm}$ for male and $>88 \mathrm{~cm}$ for female as high waist circumference, it was noticed that $68.25 \%(86)$ of the cohort studied were normal while $31.75 \%(40)$ were abnormal (Figure 1). Of those with large waist circumference, 2 were male that is $3.92 \%$ of male population, while 38 were female that is $50.67 \%$ of female population had large waist circumference.

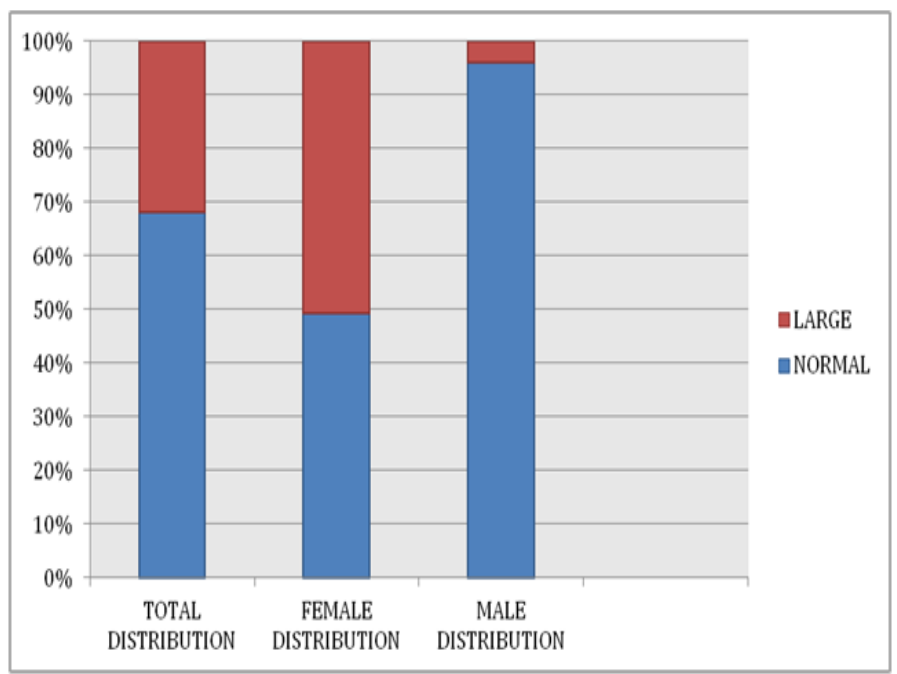

Figure I American waist circumference cut offs applied to Africans.

By using the European waist circumference cut off of $94 \mathrm{~cm}$ and above for male and $80 \mathrm{~cm}$ and above for female as high waist circumference it was noticed that $43.45 \%(55)$ of the cohorts studied were normal while $56.35 \%(71)$ were abnormal (Figure 2).

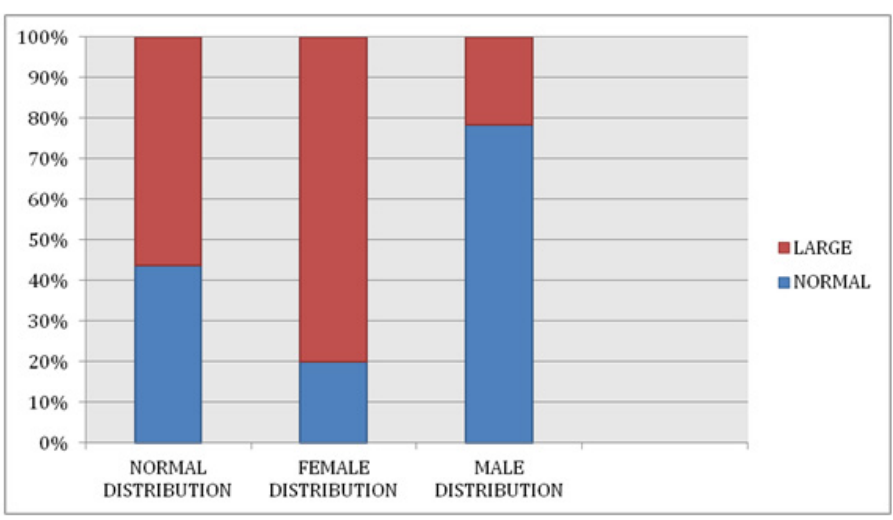

Figure 2 European waist circumference cut offs applied to Africans.

Of those with large waist circumference, 11 were male that is $21.58 \%$ of male population, while 60 were female that is $80 \%$ of the female population had large waist circumference. By using the Asian waist circumference cut off of greater than $90 \mathrm{~cm}$ in male and greater than $80 \mathrm{~cm}$ in female as high waist circumference, it was noticed that $43.65 \%(55)$ of the cohort studied were normal while $56.35 \%(71)$ were abnormal (Figure 3). Of those with large waist circumference, 18 were male that is $35.29 \%$ of male population while 53 were female that is $70.67 \%$ of female population had large waist circumference. In this cohort, the mean waist circumference in female was $89.25 \mathrm{~cm}$ while the mean waist circumference in male was $80.55 \mathrm{~cm}$.

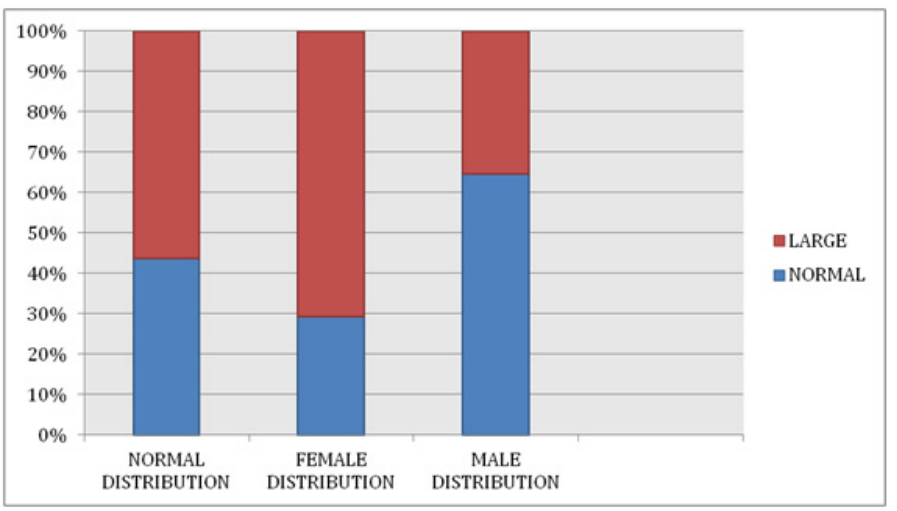

Figure 3 Asian waist circumference cut offs applied to Africans.

\section{Discussion}

The disparity in using the different cut off for Africa is glaring. Both Asian and European cut off gave same percentage of abnormal (high) waist circumference in the general population $56.35 \%$ in African, but with disparity genderwise, the Asian cut off included more males but lesser females among the African cohorts studied.

The difference in cut off for female using Asian and European cut off is less than one, yet it translated to a difference of $10 \%$ in the female African population with large waist circumference, hence using other races cut off will definitely bring about error in diagnosis in Africans. Since the sensitivity, specificity and predictive value of these other races cut offs is/are not known in African. African definitely needs its own waist circumference cut offs.

A closer look at this cohort shows a mean waist circumference for female of $89.25 \mathrm{~cm}$, if this value is taken as the normal waist circumference for these African cohorts, then we will see that it is close to the American cut off of $88 \mathrm{~cm}$ for female. Furthermore, it 
defines $49.33 \%$ (37) of the female population as having large waist circumference. The mean male waist circumference for this cohort of 80.55 if taken as normal for this cohort, defines $50.98 \%$ (26) of the male population as having large waist circumference. Though this cut off of $80.55 \%$ is markedly far from the American, European or Asian cut off for male. The male sample in this study is probably not representative of the male African population.

The Americans (USA) uses a waist circumference of $102 \mathrm{~cm}$ or less for male and $88 \mathrm{~cm}$ or less for female as normal values for their population. The National Heart, Lung, and Blood Institute's/ North American Association for the Study of Obesity committee also recommends using waist circumference cut points of 40 inches $(102 \mathrm{~cm})$ in men and 35 inches $(88 \mathrm{~cm})$ in women to define "central obesity." Increasingly, research shows that WC or central obesity is a better predictor of chronic diseases, mainly type 2 diabetes, hypertension and dyslipidemia, than overall adiposity assessed using body mass index (BMI), ${ }^{6,7} \mathrm{WC}$ may be equally or more useful than BMI due to its higher predictive value for future health risks, ease of measurement, and understanding by the general public. ${ }^{6-8}$

Different waist circumference or BMI cut off points may be necessary to adequately reflect risk in different racial/ethnic groups. ${ }^{9}$ The American findings indicate that the rise in adiposity in the United States was unequally distributed across the population and that shifts across the spectrum of BMI and WC varied between race/ ethnicity and gender groups. There are several patterns noted for the population-level changes in BMI and WC between the later 1980s and 2004. First, overall, both BMI and WC distribution appeared to shift (increase) faster in the upper end of the population distribution (i.e., those with high BMI and WC). In other words, heavier Americans had become heavier over time-"the fat becoming fatter," though this varied by gender and ethnicity, it is worth noting from this American findings that while American are comparing their weight and waist in times past to the present with projection for the future of Americans with complain that the heavy Americans are getting heavier. Africans cannot say objectively whether they are getting heavier (due to westernisation), thinner (due to hard work, war, internal displacement from tribal conflicts etc) or whether their weight and waist is static for any reason since Africans have no past reference point on waist circumference, no present reference value talk less of a future projection, African have to start somewhere despite our socioeconomic diversity and multi-ethnicity as today is the beginning of our tomorrow! The European uses a waist circumference of less than $94 \mathrm{~cm}$ as normal for male and measurement of less than $80 \mathrm{~cm}$ as normal in Europid women. ${ }^{10}$ The cut-off points of anthropometric indices such as BMI and waist circumference are usually obtained by epidemiological studies conducted on Caucasians, especially Europeans; therefore, they are not necessarily representative of the indices in other ethnicities. ${ }^{11,12}$

On the other hand, the Asian uses a waist circumference of $90 \mathrm{~cm}$ or less as normal in male and waist circumference of $80 \mathrm{~cm}$ or less as normal in Asian women. Biologically, there is evidence to show that Asians are more likely to develop diabetes for the same level of body mass index or waist circumference than their Caucasian counterparts. ${ }^{13}$ This is thought to be partly due to their propensity to store fat viscerally rather than subcutaneously, which is not captured in the traditional measures of adiposity such as body mass index and waist circumference. ${ }^{14}$ Whether Africans are equally more likely to develop diabetes or not at the same level of waist circumference like Caucasian or Asian counterpart is not objectively known.
Apart from genetic causes, we now recognize the field of epigenetics where environmental or 'nurture' exposures affect gene expression. For example, fetal exposure to maternal malnutrition during pregnancy may result in a fetal phenotype that promotes survival in a nutrient poor environment but substantially increases the risk of diabetes and cardiovascular-renal disease during a time of nutrient abundance. ${ }^{15}$ Asian women have a high prevalence of gestational diabetes, which is another important risk factor linked to future diabetes. ${ }^{15}$ development by the offspring.

Metabolic health in childhood has also been associated with future risk of obesity and diabetes, where childhood obesity increases the risk of future diabetes development. These factors result in increased trans-generational diabetes with increasingly early onset of disease, thus setting up a vicious cycle of 'diabetes begetting diabetes' ${ }^{16}$ This trans-generational diabetes with increasing early onset of disease needs proper detailed evaluation in African as well as a scientifically described African waist circumference phenotype especially in the female African for the purpose of further research in gestational diabetes among Africans.

\section{Conclusion}

The use of adopted cut offs for Africans within the African continent or in diaspora will bring about diagnostic error of varying degree in African subjects by either under diagnosing or over diagnosing large waist circumference since the sensitivity; specificity and predictive value of other races cut off will be different amongst African subjects. Africans need to arise to research and generate an indigenous African descent based cut offs for better prediction of cardiovascular risk amongst Africans.

\section{Acknowledgements}

None.

\section{Conflict of interest}

The author declares no conflict of interest.

\section{Funding}

This research received no grant from any organization whether governmental or non-governmental.

\section{References}

1. World health organisation. Definition of region groupings. Geneva, Switzerland: WHO; 2017.

2. Lam BC, Koh GC, Chen C, et al. Comparison of body mass index (BMI), body adiposity index (BAI), waist Circumference (WC), waistto-hip ratio (WHR) and waist-to-height ratio (WHtR) as predictors of cardiovascular disease risk factors in an adult Population in Singapore. PLoS One. 2015;10(4):e0122985.

3. Maffeis C, Pietrobelli A, Grezzani A, et al. Waist circumference and cardiovascular risk factors in prepubertal children. Obes Res. 2001;9(3):179-187.

4. Motala AA, Mbanya JC, Ramaiya KL. Metabolic syndrome in sub-Saharan Africa. Ethn Dis. 2009;19(2 Suppl 2):S2-S10.

5. Waist circumference and waist-hip ratio: report of a WHO expert consultation. Geneva, Switzerland: WHO; 2011. 39 p.

6. Wang Y, Rimm EB, Stampfer MJ, et al. Comparison of abdominal adiposity and overall obesity in predicting risk of type 2 diabetes among men. Am J Clin Nutr. 2005;81(3):555-563. 
7. Zhu S, Heymsfield SB, Toyoshima H, et al. Race-ethnicity-specific waist circumference cutoffs for identifying cardiovascular disease risk factors. Am J Clin Nutr. 2005;81(2):409-415.

8. The practical guide: Identification, Evaluation, and Treatment of Overweight and Obesity in Adults. NHLBI Obesity Education Initiative, Bethesda, USA: North American Association for the Study of Obesity (NAASO); 2000.

9. Carroll JF, Chiapa AL, Rodriquez M, et al. Visceral fat, waist circumference, and BMI: impact of race/ethnicity. Obesity (Silver Spring). 2008;16(3):600-607.

10. The IDF consensus worldwide definition of the metabolic syndrome. Obesity and metabolism. 2005;2(3):47-49.
11. Shabnam AA, Homa K, Reza MT, et al. Cut-off points of waist circumference and body mass index for detecting diabetes, hypercholesterolemia and hypertension according to national non-communicable disease risk factors surveillance in Iran. Arch Med Sci. 2012;8(4):614-621.

12. Razak F, Anand SS, Shannon H, et al. Defining obesity cut points in a multiethnic population. Circulation. 2007;115(16):2111-2118.

13. Yoon KH, Lee JH, Kim JW, et al. Epidemic obesity and type 2 diabetes in Asia. Lancet. 2006;368(9548):1681-1688.

14. Misra A, Khurana L. Obesity and the metabolic syndrome in developing countries. J Clin Endocrinol Metab. 2008;93(11 Suppl 1):S9-S30.

15. Chan JC, Yeung R, Luk A. The Asian Diabetes phenotypes: Challenges and opportunities. Diabetes Res Clin Pract. 2014;105(1):135-139.

16. Ma RC, Chan JC, Tam WH, et al. Gestational diabetes, maternal obesity, and the NCD burden. Clin Obstet Gynecol. 2013;56(3):633-641. 